\section{Schulung TARMED}

FMH-Tarifdienst
Der Ordner kann schriftlich beim Tarifdienst mittels dem in dieser Nummer publizierten Talon bestellt werden. Neu ist im Preis des Ordners auch die «online»-Aktualisierung inbegriffen. Inhaber von Schulungsordnern der ersten Auflage können sich ebenfalls beim Tarifdienst für die «online»-Aktualisierung anmelden.

\section{Schulungskurse}

Ungeachtet des Einführungstermins von TARMED sollte mit der Schulung vor Ende 2001 begonnen werden. Erst nach genauer Kenntnis der Tarifmechanik bzw. dessen korrekter Anwendung ist der Tarifanwender in der Lage, die Auswirkungen von TARMED auf seine Praxis abzuschätzen.

Die FMH empfiehlt allen Mitgliedern, nur Kurse von FMH-anerkannten Instruktorinnen und Instruktoren $\mathrm{zu}$ besuchen. An diesen Kursen wird die korrekte Anwendung von TARMED vermittelt.

Nebst den Instruktoren aus kantonalen Gesellschaften und Fachgesellschaften wurden auch ein beschränkte Zahl von nicht-ärztlichen Instruktoren ausgebildet. Diese Instruktoren bieten neben den FMH-eigenen auch entsprechende Schulungen an. Diese Instruktoren vermitteln die Handhabung der Tarifstruktur gemäss den Instruktionen der FMH. Für die übrigen Bestandteile der Seminare kann die FMH keine Verantwortung übernehmen.

\section{Schulungsunterlagen}

Als Schulungsunterlage hat der Tarifdienst für die FMH-Mitglieder einen Ordner zusammengestellt. Der Schulungsordner gibt zusammen mit einer CD-ROM einen detaillierten Überblick über TARMED. Die wichtigsten Positionen des Tarifes werden einzeln erklärt. Zusammen mit Fallbeispielen, welche pro Fachgebiet laufend ergänzt werden, bietet der Schulungsordner eine solide Grundlage für das Erlernen des neuen Tarifes. Der aktuelle Schulungsordner richtet sich in erster Linie an in der Grundversorgung tätige Ärztinnen und Ärzte. Für Spitalärztinnen und -ärzte wird ein eigener Ordner zusammengestellt werden.

Der Aktualisierung des Ordners wird durch den FMH-Tarifdienst sichergestellt. Um eine schnelle Aktualisierung zu einem möglichst günstigen Preis zu gewährleisten, erfolgt der Versand ausschliesslich auf elektronischem Weg. Die Besteller der Ordner müssen einzig über eine E-mail-Adresse verfügen.

\section{Informationen auf Internet}

Weitere Informationen zu TARMED findet man im Internet unter www.tarmed.ch. Es handelt sich dabei um generelle Informationen und nicht um standespolitische Stellungnahmen.

Spezifische, FMH-interne Informationen sind in einer Closed User Group (CUG) auf www.tarmed.ch publiziert. In der CUG sind u. a. diverse Abfragen aus der TARMED-Datenbank publiziert. Da diese Informationen nicht für alle FMH-Mitglieder von gleicher Bedeutung sind, ist der Zugang zur CUG nicht durch die allgemeinen Mitgliedergebühren finanziert, sondern kostenpflichtig.

\section{La version française suivra}




\section{Bestellung Schulungsordner TARMED}

2. ergänzte Auflage

Der Schulungsordner ist die Basis aller offiziellen TARMED-Schulungskurse.

Inhalt des Schulungsordners

- CD-ROM mit Tarifbrowser 1.0 (PC und Mac); Sammlung aller Fragen zu TARMED;

- Grundlagen des Tarifes;

- Anwendung der wichtigsten Leistungen;

- Fallbeispiele der Grundversorgung;

- Dignitätskonzept.
Der Ordner ist modular aufgebaut. Dies ermöglicht die laufende Ergänzung mit Fallbeispielen aller Fachgebiete. Der Upgrade des Inhaltes erfolgt aus Kostengründen ausschliesslich per E-mail.

Der Preis für Schulungsordner, CD-ROM und regelmässige Upgradings beträgt Fr. 72.50 (inkl. Portound Versandspesen). Die Bestellung kann schriftlich oder per Fax an folgende Bestelladresse gerichtet werden:

FMH-Tarifdienst, Postfach 246, 6208 Oberkirch, Fax 0419210586

Aus organisatorischen Gründen kann der Versand der Ordner erst nach Zahlungseingang erfolgen!

\section{Bestelltalon}

Ich bestelle hiermit Stück des TARMED-Schulungsordners à Fr. 72.50

Name / Vorname

Adresse

PLZ / Ort

E-mail-Adresse

Unterschrift 